\title{
Planar Rectifiable Curves Are Determined by Their Projections
}

\section{T. J. Richardson}

AT\&T Bell Laboratories, Room 2C-370, Murray Hill, NJ 07974, USA

tjr@bell-labs.com

\begin{abstract}
In 1954 Steinhaus raised the question of whether a rectifiable curve is characterized by its projections. A projection onto a line $G$ at the point $p$ counts the number of points in the set which lie on the line which is perpendicular to $G$ and passes through $p$. We prove this is so, and give a method to reconstruct a closed connected rectifiable set from its projections.
\end{abstract}

\section{Introduction}

A fundamental inverse problem in integral geometry is to determine when sets are determined by its projections. A projection onto a line $G$ at the point $p$ counts the number of points in the set which lie on the line which passes through $p$ and is perpendicular to $G$. A classical version of this inverse problem, the subject of this paper, is whether (the trace of) a rectifiable curve $A$ is determined by its projections. This problem was raised by Steinhaus [11] in 1954. (Steinhaus actually said "We believe without proof this theorem to be true.") This paper answers the problem in the affirmative by giving a method for reconstructing the curve $A$ from knowledge of the projections of $A$. Some history and results on the problem appear as problem G8 in [1]. Santaló [9] indicates a proof for rectifiable Jordan curves. Fast [3] independently solved Steinhaus's problem. The results in [3] are actually more general, allowing recovery of closed (not necessarily connected) sets. However, the method of reconstruction presented in this paper is simpler and more direct than that in [3].

In an earlier paper [8] we solved a similar uniqueness and reconstruction problem over the class of sets of finite total absolute curvature, which we call K-sets. However, not all rectifiable curves trace out a set of this type. This paper is based on a reconstruction method different from that of [8], but it is related to it in that both reconstruction methods are founded on differentiability properties of the projections. The reconstruction method of this paper is both more complicated and less stable than the reconstruction method 
for K-sets, in cases where both methods apply. The two reconstruction methods are independent in the sense that each method applies to some set that the other does not. (K-sets can have multiplicities.)

\section{Main Result}

Let $\mathbb{H}$ denote the set of lines in the plane and let $\mathbb{H}^{\text {or }}$ denote the set of oriented lines. Let $\mathbb{S}^{1}$ denote the unit sphere in $\mathbb{R}^{2}$. An oriented line $H^{\text {or }}$ in the plane is represented by $(p, \theta) \in \mathbb{R} \times \mathbb{S}^{1}$, where $\theta$ is the positively oriented unit tangent to the corresponding line $\mathcal{O}\left(H^{\text {or }}\right):=\left\{x \in \mathbb{R}^{2}:\langle x, * \theta\rangle=p\right\}$, where $* \theta \in \mathbb{S}^{1}$ denotes the unit vector obtained by rotating $\theta$ through (positive) $90^{\circ}$. Here $\mathcal{O}: \mathbb{H}^{\text {or }} \rightarrow \mathbb{H}$ represents the two sheeted covering of $\mathbb{H}$ by $\mathbb{H}^{\text {or }}$ and $\langle\cdot, \cdot\rangle$ denotes the standard inner product in $\mathbb{R}^{2}$. The measure $d \mu:=d p d \theta$ is the kinematic density on $\mathbb{H}^{\text {or }}$ [9]. The kinematic density is the Haar measure (i.e., the invariant measure) on $\mathbb{H}$ induced by Euclidean motion in the plane. The parameters $p$ and $\theta$ serve as local coordinates on $\mathbb{H}$ via $\mathcal{O}$. Hence, the kinematic density on $\mathbb{H}$ is locally expressible as $d p d \theta$.

To a set $A$ in the plane one can associate a function $n_{A}: \mathbb{H} \rightarrow \overline{\mathbb{Z}^{+}}$, defined by $n_{A}(H):=\mathcal{H}^{0}(A \cap H)\left(\mathcal{H}^{0}\right.$ is the counting measure. $)$ This function is referred to as the characteristic Crofton function [6] of the set $A$. If we fix $\theta$, then the function $n_{A}(p, \theta)$, defined for $p \in \mathbb{R}$, is the projection of $A$ in the direction $\theta$. For notational simplicity, we use $\tilde{n}_{A}: \mathbb{H}^{\text {or }} \rightarrow \overline{\mathbb{Z}^{+}}$to denote $n_{A} \circ \mathcal{O}$.

The Favard, or integral geometric, measure [4], [5], [7], [10] of a Borel set $B$ is defined as

$$
\mathcal{I}(B):=\frac{1}{2} \int_{\mathbb{H}} n_{B}(H) d \mu .
$$

The functional $\mathcal{I}$ extends to a Borel regular measure. A fundamental theorem of integral geometry is Crofton's formula,

$$
\text { length }(c)=\mathcal{I}(c) \text {, }
$$

where $c$ is a curve. More generally, the one-dimensional Hausdorff measure [2], [5], [10], [12], $\mathcal{H}^{1}$, defined by

$$
\mathcal{H}^{1}(A)=\lim _{\delta \rightarrow 0} \inf \left\{\sum_{i=1}^{\infty} \operatorname{diam}\left(U_{i}\right): A \subset \bigcup_{i=1}^{\infty} U_{i}, \operatorname{diam}\left(U_{i}\right) \leq \delta\right\},
$$

agrees with $\mathcal{I}$ on rectifiable sets [5, (3.2.26)], [10].

Steinhaus defines the following distance function for plane sets:

$$
d_{\mathcal{I}}\left(A_{1}, A_{2}\right)=\int_{\mathbb{H}}\left|n_{A_{1}}(H)-n_{A_{2}}(H)\right| d \mu .
$$

The problem Steinhaus [11] raised was whether $d_{\mathcal{I}}$ is a metric on $\mathcal{H}^{1}$ equivalence classes. Obviously, $d_{\mathcal{I}}(\cdot, \cdot) \geq 0$ and $d_{\mathcal{I}}$ satisfies the triangle inequality. Thus, Steinhaus' question is whether $d_{\mathcal{I}}\left(A_{1}, A_{2}\right)=0$ implies $\mathcal{H}^{1}\left(A_{1} \Delta A_{2}\right)=0$, where $\triangle$ denotes symmetric difference. There are entirely nonrectifiable sets of positive and finite $\mathcal{H}^{1}$ measure. Such 
sets have zero Favard measure [2], [5]. Every set of finite $\mathcal{H}^{1}$ measure uniquely decomposes, modulo sets of $\mathcal{H}^{1}$ measure zero, into a rectifiable part and entirely nonrectifiable part. Thus, apart from the issue of multiplicity, the most general version of Steinhaus's problem concerns rectifiable sets.

In this paper we prove the following.

Theorem 2.1. If $A_{1}$ and $A_{2}$ are closed connected rectifiable sets, then $d_{\mathcal{I}}\left(A_{1}, A_{2}\right)=0$ if and only if $\mathcal{H}^{1}\left(A_{1} \triangle A_{2}\right)=0$.

One direction is obvious: $d_{\mathcal{I}}\left(A_{1}, A_{2}\right)=0$ if $\mathcal{H}^{1}\left(A_{1} \Delta A_{2}\right)=0$ since $\mathcal{I}$ and $\mathcal{H}^{1}$ agree on rectifiable sets. We prove the opposite implication by giving a procedure to reconstruct $A$ from any measurable function $\hat{n}$ satisfying $\int_{\mathbb{H}}\left|n_{A}(H)-\hat{n}(H)\right| d \mu=0$, i.e., from any representative of the $L^{1}(\mathbb{H})$ equivalence class of $n_{A}$.

In [8] it is shown that the characteristic Crofton function of sets of finite total curvature is a function of bounded variation. The theory of functions of bounded variation is exploited there as the basis of the reconstruction. For less regular sets, however, the characteristic Crofton function has a less accessible structure and a different approach is required.

Gelfand and Smirnov [6] define the concept of a Crofton density. An even 1-density in $\mathbb{R}^{2}$ is a real-valued function $\varphi(x, u)$ of $x \in \mathbb{R}^{2}$ and a vector $u$ in the tangent space at $x$ such that

$$
\varphi(x, a u)=|a| \varphi(x, u)
$$

for all $a \in \mathbb{R}$. (To simplify the notation we identify the tangent space to $x$ in $\mathbb{R}^{2}$ with $\mathbb{R}^{2}$ in the canonical way, i.e., $u \in \mathbb{R}^{2}$.) Even densities can be integrated over manifolds without orientation [6]. An even 1-density $\varphi$ is a Crofton 1-density in $\mathbb{R}^{2}$ if the integral of $\varphi$ over a manifold can be represented as the integral of the characteristic Crofton function of the manifold with respect to a measure that is absolutely continuous with respect to the kinematic density. The reconstruction method developed for K-sets in [8] used certain simple Crofton densities. The reconstruction method of this paper considers instead more complicated Crofton densities and shows that the underlying set can be recovered from evaluation of the integrals of these Crofton densities over the set.

\section{Rectifiable Sets}

A (parametrized plane) curve is a continuous function $c:[a, b] \rightarrow \mathbb{R}^{2}$ that is nonconstant on any open subinterval. The trace of a curve $c$ is the point set image of $c$, i.e.,

$$
\operatorname{tr}(c):=\left\{x: c^{-1}(x) \neq \emptyset\right\}
$$

The length of a curve is defined by

$$
L(c):=\sup \left\{\sum_{i=1}^{n}\left|c\left(t_{i}\right)-c\left(t_{i-1}\right)\right|: a \leq t_{0}<t_{1}<\cdots<t_{n} \leq b\right\} .
$$

For $C^{1}$ curves, $L(c)$ is equal to $\int_{a}^{b}|\dot{x}(t)| d t$, i.e., the length of $c$ according to the classical definition. 
If $L(c)<\infty$, then $c$ is a rectifiable curve. A set $E \subset \mathbb{R}^{2}$ satisfying $\mathcal{H}^{1}(E)<\infty$ is rectifiable if $\mathcal{H}^{1}\left(E \backslash \bigcup_{i=1}^{\infty} \operatorname{tr} c_{i}\right)=0$ for some countable collection of rectifiable curves $\left\{c_{i}\right\}$.

Let $B_{\rho}(x)$ denote the open ball of radius $\rho$ centered at $x$, and let $B_{\rho}$ abbreviate $B_{\rho}(0)$. Let $E \subset \mathbb{R}^{n}$. Assuming it exists, the one-dimensional density of $E$ at $x$ is

$$
\Theta(E, x):=\lim _{\rho \rightarrow 0_{+}} \frac{\mathcal{H}^{1}\left(B_{\rho}(x) \cap E\right)}{2 \rho} .
$$

Points $x \in \mathbb{R}^{n}$ such that $\Theta(E, x)=1$ are regular points of $E$. If $E$ is rectifiable, then $\mathcal{H}^{1}$-almost every point in $E$ is regular [2]. Rectifiable sets also have tangents (in a suitably weak sense) $\mathcal{H}^{1}$-almost everywhere. (See [2] or (3.2.19) of [5].) Thus, even 1-densities can be integrated (with respect to $\mathcal{H}^{1}$ ) over rectifiable sets.

In this paper the symbol $v$ always denotes a unit normal to a rectifiable set $A$. A rectifiable set $A$ is oriented by $\left(\mathcal{H}^{1}\right.$-measurably) selecting a unit normal vector at each point of $A$ possessing a tangent line. For an even 1-density $\varphi$, we define

$$
\int_{A} \varphi:=\int_{A} \varphi(x, v(x)) d \mathcal{H}^{1}(x) .
$$

\section{The Reconstruction}

The reconstruction has two distinct steps. The first step finds all straight line segments in $A$. The second step yields a dense subset of that part of $A$ not containing any straight line segments. Both steps rely on using back-projection to define Crofton densities.

If $\theta_{1} \neq-\theta_{2}$, then let $\angle\left(\theta_{1}, \theta_{2}\right)$ denote the (signed) angle of minimal magnitude through which $\theta_{1}$ can be rotated to make it coincide with $\theta_{2}$. For $x \in \mathbb{R}^{2} \backslash 0$, let $\vec{x}$ denote the projection of $x$ on $\mathbb{S}^{1}$, i.e., $\vec{x}=x /|x|$.

\subsection{Weighted Back Projection}

For any $x \in \mathbb{R}^{2}$, the back projection of a rectifiable set $A$ at $x$ is essentially the total angle subtended by $A$ at $x$. Here we consider weighted back projections.

Let $T_{z}$ denote translation by $z$ in the plane. For any 1 -density $\varphi$, the 1 -density $T_{z} \varphi$ is given by $T_{z} \varphi(x, u)=\varphi\left(x-z, d T_{z}(u)\right)=\varphi(x-z, u)$.

Let $\omega \in C^{\infty}\left(\mathbb{S}^{1}\right)$ be an even function, i.e., $\omega(\theta)=\omega(-\theta)$. For each such $\omega$, we define a 1-density $\varphi^{\omega}$ as follows: set $\varphi^{\omega}(0, u)=0$ and if $x \neq 0$, then set

$$
\varphi^{\omega}(x, u):=\omega(\vec{x}) \frac{|\langle u, \vec{x}\rangle|}{|x|} .
$$

For each $x, h, u \in \mathbb{R}^{2}$ let $s(x, u, h) \in\{1,0,-1\}$ be defined as follows:

$$
s(x, u, h):= \begin{cases}\operatorname{sgn}\langle x, u\rangle, & \langle x, u\rangle \neq 0, \\ \operatorname{sgn}\langle-h, u\rangle, & \langle x, u\rangle=0 .\end{cases}
$$

For $x \neq 0$ and $u \in \mathbb{R}^{2}$, we define

$D_{h} \varphi^{\omega}(x, u):=s(x, u, h)|x|^{-2}(\omega(\vec{x})(2\langle u, \vec{x}\rangle\langle h, \vec{x}\rangle-\langle u, h\rangle)+\dot{\omega}(\vec{x})\langle u, \vec{x}\rangle\langle * h, \vec{x}\rangle)$, 
where $\dot{\omega}$ denotes the derivative of $\omega$ on $\mathbb{S}^{1}$ in the usual sense. For $x, y \in \mathbb{R}^{2}$, let

$$
\mathcal{P}(x, y):=\left\{z \in \mathbb{R}^{2}:\langle z, y\rangle\langle z, x\rangle<0\right\} .
$$

The following regularity estimate is an important device in our reconstruction.

Lemma 4.1. Let $x \in \mathbb{R}^{2} \backslash 0$ and assume $|h| \leq 0.1|x|$. Then

$$
T_{h} \varphi^{\omega}(x, u)-\varphi^{\omega}(x, u)=D_{h} \varphi^{\omega}(x, u)+e(\omega, h, x, u),
$$

where, if $u \in \mathcal{P}(x-h, x)$, then $e(\omega, h, x, u)$ satisfies

$$
|e(\omega, h, x, u)| \leq \frac{|u||h|}{|x|^{3}}\left(5|x|\|\omega\|_{\infty}+|h|\|\dot{\omega}\|_{\infty}\right),
$$

and, if $u \notin \mathcal{P}(x-h, x)$, then $e(\omega, h, x, u)$ satisfies

$$
|e(\omega, h, x, u)| \leq 6 \frac{|u||h|^{2}}{|x|^{3}}\left(\|\omega\|_{\infty}+\|\dot{\omega}\|_{\infty}+|x|\|\ddot{\omega}\|_{\infty}\right) .
$$

Proof. The proof is elementary and is given in the Appendix.

Observe that $\int_{A} T_{x} \varphi^{\omega}$ is continuous on $\mathbb{R}^{2} \backslash \bar{A}$ as a function of $x$ (here $\bar{A}$ denotes the topological closure of $A$.) There may be points $x \in \bar{A}$ at which $\int_{A} T_{x} \varphi^{\omega}$ does not have a continuous extension. Rather than concern ourselves with these points, we smooth $\varphi^{\omega}$. For any $x, u \in \mathbb{R}^{2}$ and $\rho>0$ define

$$
\varphi_{\rho}^{\omega}(x, u):=\frac{1}{\pi \rho^{2}} \int_{B_{\rho}(x)} \varphi^{\omega}(y, u) d y .
$$

Although $\varphi^{\omega}$ is not a Crofton density (see below), $\varphi_{\rho}^{\omega}$ is.

Lemma 4.2. For every $\rho>0, \varphi_{\rho}^{\omega}$ is a Crofton density.

Proof. If $\operatorname{dist}(0, A)>0$, then polar projection of $\mathbb{R}^{2} \backslash 0$ onto the unit circle $(x \rightarrow x /|x|)$ is a Lipschitz function on $A$. In this case we can apply the area-coarea formula of geometric measure theory [5, (3.2.22)], [7] to obtain

$$
\int_{A} \varphi^{\omega}=\frac{1}{2} \int_{\mathbb{S}^{1}} \omega(\theta) \tilde{n}_{A}(0, \theta) d \theta .
$$

Equation (4.1) shows that $\int_{A} \varphi^{\omega}$ is just the $\omega$-weighted back projection of $A$ at the origin. Strictly speaking, $\varphi^{\omega}$ is not a Crofton density because the required measure is singular with respect to the kinematic density.

Let $\eta \in C^{\infty}(\mathbb{R})$ be a nonnegative function with range $[0,1]$ and support in $[-1,1]$ satisfying $\eta(t)=1$ for $|t| \leq \frac{1}{2}$. For $r>0$, define $\varphi^{\omega, r}(x, u):=(1-\eta(r|x|)) \varphi^{\omega}(x, u)$ and $\varphi_{\rho}^{\omega, r}:=\left(1 / \pi \rho^{2}\right) \int_{B_{\rho}(x)} \varphi^{\omega, r}(y, u) d y$. Furthermore, let $n_{A, r}(H):=\int_{A \cap H}(1-$ $\eta(r|x|)) d \mathcal{H}^{0}(x)$. This function can be interpreted as the characteristic Crofton function 
of the set $A$, where the multiplicity of $A$ at $x \in A$ has been set to $1-\eta(r|x|)$. Since $\int_{A} \varphi^{\omega, r}=\int_{A \backslash B_{1 /(2 r)}} \varphi^{\omega, r}$, we can again apply the area-coarea formula (even if $0 \in A$ ) to obtain

$$
\int_{A} \varphi^{\omega, r}=\frac{1}{2} \int_{\mathbb{S}^{1}} \omega(\theta) \tilde{n}_{A, r}(0, \theta) d \theta .
$$

It follows from the Fubini theorem that

$$
\int_{A} \varphi_{\rho}^{\omega, r}=\frac{1}{2 \pi \rho^{2}} \int_{B_{\rho}} \int_{\mathbb{S}^{1}} \omega(\theta) \tilde{n}_{T_{y} A, r}(0, \theta) d \theta d y
$$

A straightforward application of the monotone convergence theorem (let $r \rightarrow \infty$ ) and a change of variables yields

$$
\begin{aligned}
\int_{A} \varphi_{\rho}^{\omega} & =\frac{1}{2 \pi \rho^{2}} \int_{B_{\rho}} \int_{\mathbb{S}^{1}} \omega(\theta) \tilde{n}_{T_{y} A}(0, \theta) d \theta d y \\
& =\frac{1}{\pi \rho^{2}} \int_{-\rho}^{\rho} \int_{\mathbb{S}^{:}} \omega(\theta)\left(\rho^{2}-p^{2}\right)^{1 / 2} \tilde{n}_{A}(p, \theta) d \theta d p
\end{aligned}
$$

\subsection{Recovering Straight Line Segments}

To recover line segments we set $\omega(\theta)=1$. Henceforth in this section we drop $\omega$ from our notation. Thus, $\varphi=\varphi^{\omega}$ where $\omega \equiv 1$.

Assume now that $A$ is a rectifiable set with bounded support, i.e., $\bar{A} \subset B_{R}$ for some $R<\infty$. It is clear that a suitable $R$ can be determined from the $L^{1}$ equivalence class of $n_{A}$ since $n_{A}(H)=0$ for all $H$ such that $B_{R} \cap H=\emptyset$. Thus, there is no loss in generality in assuming a fixed $R$.

Let $x \in \mathbb{R}^{2} \backslash \overline{B_{R}}$ and define $L_{x}(A):=\{y \in A:\langle v(y), y-x\rangle=0\}$. We conclude from (4.1) that $\int_{A} T_{x} \varphi=\lim _{\rho \rightarrow 0+} \int_{A} T_{x}\left(\varphi_{\rho}\right)$, hence $\int_{A} T_{x} \varphi$ is uniquely determined by the $L^{1}$ equivalence of $n_{A}$. Now, for any $|x|>R$, we have

$$
\int_{A} T_{x} D_{h} \varphi=\int_{T_{-x} A} D_{h} \varphi
$$

Since $\bigcap_{\varepsilon>0}\left\{y \in T_{-x} A: v(y) \in \mathcal{P}(y-\varepsilon h, y)\right\}=\emptyset$, we have

$$
\lim _{\varepsilon \rightarrow 0_{+}} \mathcal{H}^{1}\left\{y \in T_{-x} A: v(y) \in \mathcal{P}(y-\varepsilon h, y)\right\}=0
$$

and we can apply Lemma 4.1 to obtain

$$
\int_{A} T_{x} D_{h} \varphi=\lim _{\varepsilon \rightarrow 0_{+}} \varepsilon^{-1}\left(\int_{A} T_{x+\varepsilon h} \varphi-\int_{A} T_{x} \varphi\right)
$$

Thus, $\nabla_{x} \int_{A} T_{x} \varphi$ exists if and only if $\mathcal{H}^{1}\left(L_{x}(A)\right)=0$. More precisely,

$$
\int_{A} D_{h} T_{x} \varphi+\int_{A} D_{-h} T_{x} \varphi=2 \int_{L_{x}(A)} \frac{|\langle\nu, h\rangle|}{|y-x|^{2}} d \mathcal{H}^{1}(y)
$$


Since $\mathcal{H}^{1}(A)<\infty$, there are at most countably many lines $H$ for which $\mathcal{H}^{1}(H \cap A)$ is positive. It is now evident that this set of lines is determined by $\int_{A} T_{x} \varphi, x \in \mathbb{R}^{2} \backslash B_{R}$, and that if $x \in H$, then $\int_{H \cap A}|y-x|^{-2} d \mathcal{H}^{1}(y)$ is determined. By differentiating with respect to $x$ on $H$, we can also determine $\int_{H \cap A}|y-x|^{-(2+i)} d \mathcal{H}^{1}(y)$ for $i \in \mathbb{Z}^{+}$. From the StoneWeierstrass theorem and the fact that dist $(x, A)>0$, we see that $\int_{H \cap A} f(|y-x|) d \mathcal{H}^{1}(y)$ is determined for each $f \in C(\mathbb{R})$. It follows from the general theory of rectifiable sets that $A \cap H$ is uniquely determined up to a set of $\mathcal{H}^{1}$ measure zero.

Let $L_{A}$ denote the closure of the set $\{y: \Theta(A \cap H, y)=1\}$ for some $H \in \mathbb{H}$. Obviously, $L_{A} \subset A$ when $A$ is closed. We have proved the following.

Theorem 4.3. If $A$ is a rectifiable set of compact support, then $L_{A}$ is uniquely determined by the $L^{1}$ equivalence class of $n_{A}$.

\subsection{Recovering Curved Segments}

In this section we prove Theorem 2.1. The key idea is to study the regularity of $n_{A}$ near lines which are, in some sense, tangent to $A$.

Let $\tilde{\omega} \in C^{\infty}(\mathbb{R})$ be a nonnegative even function with support in $(-1,1)$ satisfying $\int_{\mathbb{R}} \tilde{\omega}(x) d x=\frac{1}{2}$ and $\tilde{\omega}(x)=0$ in some neighborhood of zero. For any $0<\varepsilon \leq 1$ and $\psi \in \mathbb{S}^{1}$, we define an even function $\omega_{\varepsilon, \psi} \in C^{\infty}\left(\mathbb{S}^{1}\right)$ by

$$
\omega_{\varepsilon, \psi}(\theta):=\varepsilon^{-1 / 2} \tilde{\omega}\left(\varepsilon^{-1 / 2} \arcsin \langle\theta, \psi\rangle\right),
$$

where $\arcsin :[-1,1] \rightarrow(-\pi / 2, \pi / 2]$. We have, $\int_{\mathbb{S}^{1}} \omega_{\varepsilon, \psi}(\theta) d \theta=1$. Let

$$
Q_{A}(x):=\sup _{\psi \in \mathbb{S}^{\prime}, s>0} \limsup _{\varepsilon \rightarrow 0_{+}}\left|\int_{A} T_{x+\varepsilon s \psi} \varphi_{\varepsilon}^{\omega_{\varepsilon}, \psi}-\int_{A} T_{x-\varepsilon s \psi} \varphi_{\varepsilon}^{\omega_{\varepsilon}, \psi}\right| .
$$

The function $Q_{A}$ is carefully designed to test for a particular regularity property of $n_{A}$. Since $T_{z} \varphi_{\varepsilon}^{\omega_{\varepsilon}, \psi}$ is a Crofton density for any $z, Q_{A}$ is determined by the $L^{1}$ equivalence class of $n_{A}$.

Lemma 4.4. Let $A$ be a rectifiable set. If $\operatorname{dist}(x, A)>0$, then $Q_{A}(x)=0$.

Proof. Fix $x, s$, and $\psi$. Without loss of generality, we can assume $x=0$. Let $\delta$ denote $\min (\operatorname{dist}(0, A), 1)$ and assume $\delta>0$. Define

$$
A_{\varepsilon}:=\left\{y \in A: \omega_{\varepsilon \cdot \psi}(\vec{y}) \neq 0\right\} .
$$

Since $\bigcap_{\xi>0}\left(\bigcup_{\varepsilon<\xi} A_{\varepsilon}\right)=\emptyset$, we have $\lim _{\varepsilon \rightarrow 0_{+}} \mathcal{H}^{1}\left(A_{\varepsilon}\right)=0$. Let $z \in B_{\varepsilon}$ be arbitrary and let $h$ denote $z+\varepsilon s \psi$. Using the notation of Lemma 4.1 we write

$$
\begin{aligned}
T_{h} \varphi^{\omega_{\varepsilon, \psi}}(y, u) & -T_{-h} \varphi^{\omega_{\varepsilon, \psi}}(y, u) \\
& =D_{h} \varphi^{\omega_{\varepsilon, \psi}}(y, u)-D_{-h} \varphi^{\omega_{\varepsilon, \psi}}(y, u)+e\left(\omega_{\varepsilon, \psi}, h, y, u\right)-e\left(\omega_{\varepsilon, \psi},-h, y, u\right) .
\end{aligned}
$$

From the estimates of Lemma 4.1 we conclude that there are constants $C_{1}=C_{1}(s, \delta)<$ 
$\infty$ and $C_{2}=C_{2}(s, \delta)<\infty$ such that if $\varepsilon(1+s)<0.1 \delta$, then

$$
\begin{aligned}
& \left|\int_{A} T_{h} \varphi^{\omega_{s, \psi}}-\int_{A} T_{-h} \varphi^{\omega_{\varepsilon, \psi}}\right| \\
& \quad \leq C_{1}(\delta, s)\left(\varepsilon^{1 / 2}\|\tilde{\omega}\|_{\infty}+\|\dot{\tilde{\omega}}\|_{\infty}\right) \mathcal{H}^{1}\left(A_{\varepsilon}\right) \\
& \quad+C_{2}(\delta, s) \varepsilon^{1 / 2}\left(\|\tilde{\omega}\|_{\infty}+\|\dot{\tilde{\omega}}\|_{\infty}+\|\tilde{\tilde{\omega}}\|_{\infty}\right) \mathcal{H}^{1}(A) .
\end{aligned}
$$

The lemma follows easily from this.

The remainder of the proof rests on establishing that $Q_{A}>0$ on a dense subset of the curved part of $A$. Our approach relies on certain properties of convex sets and functions on $\mathbb{S}^{l}$ of bounded variation.

The total variation $V_{\mathbb{S}^{1}}(g)$ of a real function $g \in L^{1}\left(\mathbb{S}^{1}\right)$ is defined as the supremum of $\left\{\sum_{i=0}^{n}\left|g\left(\theta_{i}\right)-g\left(\theta_{i-1}\right)\right|\right\}$, where $\theta_{0}, \theta_{1}, \ldots, \theta_{n}=\theta_{-1}$ is an ordered cycle of Lebesgue points [12] of $g$ in $\mathbb{S}^{1}$. If $V_{\mathbb{S}^{1}}(g)<\infty$, then we say $g \in B V\left(\mathbb{S}^{1}\right)$. Standard theory on $B V$ functions implies that if $g \in B V\left(\mathbb{S}^{1}\right)$, then $\dot{g}$ exists almost everywhere in $\mathbb{S}^{1}$.

Let $D$ be a closed bounded convex set in $\mathbb{R}^{2}$. For each $\theta \in \mathbb{S}^{1}$ define

$$
f(\theta):=\sup \{\langle x, \theta\rangle: x \in D\} .
$$

Proposition 4.5. The function $f(\theta)$ is absolutely continuous and $\dot{f} \in B V\left(\mathbb{S}^{1}\right)$. Furthermore, if $\dot{f}(\theta)$ exists, then $x_{e}(\theta):=f(\theta) \theta+\dot{f}(\theta) * \theta$ is the unique (extreme) point of $D$ satisfying $f(\theta)=\left\langle x_{e}(\theta), \theta\right\rangle$.

Proof. Let $\bar{f}:=\sup _{\theta} f(\theta)=\sup _{x \in D}|x|$ and assume $\bar{f}>0$. Let $\theta_{1}, \theta_{2} \in \mathbb{S}^{1}$ and assume that $f\left(\theta_{2}\right) \geq f\left(\theta_{1}\right)$. We can find $x \in \partial D$ such that $\left\langle x, \theta_{2}\right\rangle=f\left(\theta_{2}\right)$. It follows that $f\left(\theta_{1}\right) \geq\left\langle x, \theta_{1}\right\rangle$ so $f\left(\theta_{2}\right)-f\left(\theta_{1}\right) \leq|x|\left|\theta_{2}-\theta_{1}\right| \leq \bar{f}\left|\angle\left(\theta_{2}, \theta_{1}\right)\right|$. Thus, $f$ is absolutely continuous. A simple argument, which we omit, shows that $f$ has left and right derivatives, $\dot{f}\left(\theta_{-}\right)$and $\dot{f}\left(\theta_{+}\right)$respectively, for all $\theta$. Furthermore,

$$
\{x \in D:\langle x, \theta\rangle=f(\theta)\}=\{f(\theta) \theta+\beta * \theta: \beta \in[f(\theta-), f(\theta+)]\} .
$$

Therefore, if $\dot{f}(\theta)$ exists, then $\partial D \cap H_{\theta}$ is a singleton. Moreover, it follows easily that $\dot{f} \in B V\left(\mathbb{S}^{1}\right)$.

From Proposition 4.5 , we conclude that $\ddot{f}$ exists for almost all $\theta \in \mathbb{S}^{1}$. Using this fact, we obtain the following.

Lemma 4.6. For almost all $\theta \in \mathbb{S}^{1}$, there exists a unique extreme point $x_{e}(\theta)$ of $D$, satisfying $f(\theta)=\left\langle x_{e}, \theta\right\rangle$, and a real number $K(\theta)<\infty$ such that

$$
T_{x_{\varepsilon}+s \varepsilon \theta} \varphi_{\varepsilon}^{\omega_{\varepsilon, \theta}}(y, \cdot) \equiv 0
$$

for all $y \in D$ whenever $s \geq K$ and $\varepsilon \leq K^{-1}$. 
Proof. By Proposition 4.5, $\dot{f}$ and $\ddot{f}$ exist $\mathcal{H}^{1}$-almost everywhere in $\mathbb{S}^{1}$. Let $\theta$ be a point where $\dot{f}$ and $\ddot{f}$ exist, and let $\delta \in(0,1)$ be small enough so that if $|\angle(\psi, \theta)| \leq \delta$, then

$$
f(\psi) \leq f(\theta)+\dot{f}(\theta) \angle(\theta, \psi)+(1+|\ddot{f}(\theta)|)[\angle(\theta, \psi)]^{2} .
$$

By Proposition 4.5, $x_{e}=f(\theta) \theta+\dot{f}(\theta) * \theta$ is the unique point in $D$ such that $f(\theta)=$ $\left\langle x_{e}, \theta\right\rangle$. It follows that if $|\angle(\psi, \theta)| \leq \delta$, then

$$
\begin{aligned}
\left\langle x_{e}, \psi\right\rangle-f(\psi) \geq & f(\theta)(\langle\theta, \psi\rangle-1)+\dot{f}(\theta)(\langle * \theta, \psi\rangle-\angle(\theta, \psi)) \\
& -(1+|\ddot{f}(\theta)|)[\angle(\theta, \psi)]^{2} \\
\geq & -\left(\left|x_{e}\right|+1+|\ddot{f}(\theta)|\right)[\angle(\theta, \psi)]^{2} .
\end{aligned}
$$

Let $K(\theta)=\max \left\{\delta^{-2}, 2\left(\left|x_{e}\right|+2+|\ddot{f}(\theta)|\right)\right\}$ and assume $s \geq K$ and $\varepsilon \leq K^{-1}$. Let $z \in B_{\varepsilon}$ and $y \in \mathbb{R}^{2} \backslash\left(x_{e}+s \varepsilon \theta+z\right)$ be such that $T_{x_{e}+s \varepsilon \theta+i} \varphi^{\omega_{\varepsilon, \theta}}(y, u) \neq 0$ for some $u \in T_{y}$. We show that this implies $y \notin D$, which proves the lemma.

Since $T_{x_{e}+s \varepsilon \theta+z} \varphi^{\omega_{t, \theta}}(y, u) \neq 0$, the line through $y$ and $x_{e}+s \varepsilon \theta+z$ has a unit normal $\psi$ that satisfies $|\angle(\theta, \psi)| \leq \varepsilon^{\frac{1}{2}} \leq \delta$. Note that $\langle\theta, \psi\rangle>\frac{1}{2}$. Using this and (4.3) we obtain

$$
\begin{aligned}
\langle y, \psi\rangle-f(\psi) & =\left\langle x_{e}+s \varepsilon \theta+z, \psi\right\rangle-f(\psi) \\
& \geq s \varepsilon\langle\theta, \psi\rangle-\varepsilon\left(\left|x_{e}\right|+2+|\ddot{f}(\theta)|\right) \\
& >0
\end{aligned}
$$

and, therefore, $y \notin D$.

We are now ready to prove the main technical lemma.

Lemma 4.7. If $A$ is a closed connected rectifiable set, then the set $\left\{Q_{A} \geq 1\right\}$ is dense in $A \backslash L_{A}$.

Proof. Let $y \in A$ be a regular point of $A$ disjoint from $L_{A}$. Since $A$ is connected and $\mathcal{H}^{\mathrm{l}}\left(\boldsymbol{B}_{\rho}(y) \cap A\right) \geq \int_{0}^{r} \mathcal{H}^{0}\left(\partial B_{\rho}(y) \cap A\right) d \rho$, we can conclude that $\mathcal{H}^{0}\left(\partial B_{\rho}(y) \cap A\right) \in$ $\{1,2\}$ for infinitely many arbitrarily small $\rho$. Let $D_{\rho}$ denote the closed convex hull of $A \cap B_{\rho}(y)$ and assume $\mathcal{H}^{0}\left(\partial B_{\rho}(y) \cap A\right) \in\{1,2\}$. Since $y$ is disjoint from $L_{A}$, it follows that $D_{\rho}$ has a nonempty interior. Thus, there exists an open subset $E \in \mathbb{S}^{1}$ such that if $\theta \in E$ and $x \in D_{\rho}$ maximizes $\langle x, \theta\rangle$ over $D_{\rho}$, then for $x \notin \partial B_{\rho}$. By Lemma 4.6 we can find $\theta \in E$ such that there is a unique extreme point $x$ of $D_{\rho}$ with exterior normal $\theta$. Furthermore, there is a finite constant $1<K<\infty$ such that if $\varepsilon<K^{-1}$, then $\int_{A \cap B_{\rho}(y)} T_{x+\varepsilon K \theta} \varphi_{\varepsilon}^{\omega_{\varepsilon, \theta}}=0$. Since $x$ is an extreme point of $D_{\rho}$, we have $x \in A$. Since $A$ is connected, there exists a path inside $A \cap D_{\rho}$ from $x$ to some $x^{\prime} \in \partial B_{\rho} \cap D_{\rho}$. (Connected and pathwise connected are the same for rectifiable sets in the plane [2].) Since $(x, \theta\rangle\rangle\left\langle x^{\prime}, \theta\right\rangle$ it follows that if $\varepsilon$ is sufficiently small, and $T_{x-\varepsilon K \theta+z} \varphi^{\omega_{\varepsilon, \theta}}(w, u) \neq 0$, where $z \in B_{\varepsilon}$, then the line $H$ through $x-\varepsilon K \theta+z$ and $w$ intersects the line segment joining $x$ to $x^{\prime}$. Hence, $H$ intersects the path from $x$ to $x^{\prime}$ in $A \cap D_{\rho}$. We conclude

$$
\int_{A \cap B_{\rho}(y)} T_{x-\varepsilon K \theta} \varphi_{\varepsilon}^{\omega_{\varepsilon, \theta}} \geq 1
$$


From Lemma 4.4, we deduce $Q_{A \backslash B_{\rho}(y)}(x)=0$ and we now obtain $Q_{A}(x) \geq 1$. Since $\rho$ is arbitrarily small, we conclude that $y$ is in the topological closure of $\left\{Q_{A} \geq 1\right\}$.

By Lemmas 4.4 and 4.7 , we see that if $A$ is a closed connected rectifiable set, then $A=\overline{\left\{Q_{A} \geq 1\right\}} \cup L_{A}$. Since $Q_{A}(x)$ is determined by the $L^{1}$ equivalence class of $n_{A}$, we see, by Theorem 4.3 , that the proof of Theorem 2.1 is complete.

\section{Appendix}

Proof of Lemma 4.1. We assume throughout that $|h| \leq 0.1|x|$.

Assume first that $u \in \mathcal{P}(x-h, x)$. Then

$$
|\langle x, u\rangle| \leq|u|\left|\left\langle * \frac{x-h}{|x-h|}, x\right\rangle\right| \leq|u||h|
$$

and, similarly, $|\langle x-h, u\rangle| \leq|u||h|$. We now obtain the bounds

$$
\begin{aligned}
\left|T_{h} \varphi^{\omega}(x, u)-\varphi^{\omega}(x, u)\right| & \leq\|\omega\|_{\infty}\left(\frac{|\langle x-h, u\rangle|}{|x-h|^{2}}+\frac{|\langle x, u\rangle|}{|x|^{2}}\right) \\
& \leq 3\|\omega\|_{\infty}|u||h||x|^{-2} \\
\left|D_{h} \varphi^{\omega}(x, u)\right| & \leq 2\|\omega\|_{\infty}|u||h||x|^{-2}+\|\dot{\omega}\|_{\infty}|u||h|^{2}|x|^{-3} .
\end{aligned}
$$

Combining these bounds yields the claimed estimate.

Henceforth, we assume $u \notin \mathcal{P}(x-h, x)$. Since $\varphi^{\omega}(x, u)$ is an even 1-density, we can assume that $s(x, u, h)=1$ without loss of generality. We now have

$$
\begin{aligned}
T_{h} \varphi^{\omega}(x, u)-\varphi^{\omega}(x, u)= & {\left[T_{h} \omega(\vec{x})-\omega(\vec{x})\right]\left[T_{h}\left(|x|^{-2}\langle x, u\rangle\right)-|x|^{-2}\langle x, u\rangle\right] } \\
& +\left[T_{h} \omega(\vec{x})-\omega(\vec{x})\right]|x|^{-2}\langle x, u\rangle \\
& +\omega(\vec{x})\left[T_{h}\left(|x|^{-2}(x, u\rangle\right)-|x|^{-2}\langle x, u\rangle\right]
\end{aligned}
$$

The first term in (A.1) can be bounded above by $6\|\dot{\omega}\|_{\infty}|h|^{2}|u||x|^{-3}$. Since $\|\dot{\omega}\|_{\infty} \leq$ $\pi\|\ddot{\omega}\|_{\infty}$, we obtain the estimate

$$
\begin{aligned}
\mid T_{h} \omega(\vec{x})- & \omega(\vec{x})-\dot{\omega}(\vec{x}) \frac{\langle x, * h\rangle}{|x|^{2}} \mid \\
& \leq\|\dot{\omega}\|_{\infty}\left|\frac{\langle\vec{x}, * h\rangle}{|x|}-\arcsin \left(\frac{\langle\vec{x}, * h\rangle}{|x-h|}\right)\right|+\|\ddot{\omega}\|_{\infty} \arcsin ^{2}\left(\frac{\langle\vec{x}, * h\rangle}{|x-h|}\right) \\
& \leq 6|x|^{-2}\|\ddot{\omega}\|_{\infty}|h|^{2} .
\end{aligned}
$$

Let

$$
F(x, u, h):=\frac{2\langle\vec{x}, u\rangle\langle\vec{x}, h\rangle-\langle u, h\rangle}{|x|^{2}}
$$

Then

$$
T_{h}\left(\frac{\langle x, u\rangle}{|x|^{2}}\right)-\left(\frac{\langle x, u\rangle}{|x|^{2}}\right)=F(x, u, h) \frac{|x|^{2}}{|x-h|^{2}}-\frac{|h|^{2}\langle x, u\rangle}{|x|^{2}|x-h|^{2}}
$$


Since $|F(x, u, h)| \leq 2|u||h||x|^{-2}$, we obtain

$$
\left|T_{h}\left(\frac{\langle x, u\rangle}{|x|^{2}}\right)-\frac{\langle x, u\rangle}{|x|^{2}}-F(x, u, h)\right| \leq 6 \frac{|h|^{2}}{|x|^{3}} .
$$

Substituting (A.3) and (A.2) into (A.1), the desired result is obtained.

\section{References}

1. H. T. Croft, K. J. Falconer, R. K. Guy, Unsolved Problems in Geometry, Springer-Verlag, New York, 1991.

2. K. J. Falconer, The Geometry of Fractal Sets, Cambridge University Press, New York, 1985.

3. H. Fast, Inversion of the Crofton transform for sets in the plane, Real Anal. Exchange, 19(1), (1994), 59-80.

4. J. Favard, Définition de longeur et de l'aire, C. R. Acad. Sci. Paris, 194 (1932), 334-346.

5. H. Federer, Geometric Measure Theory, Springer-Verlag, New York, 1969.

6. I. M. Gelfand, M. M. Smimov, Crofton densities and nonlocal differentials, in The Gelfand Mathematical Seminars, 1990-1992, L. Corwin, I. Gelfand, J. Lepowsky, eds., Birkhäuser, Boston, 1993.

7. F. Morgan, Geometric Measure Theory: A Beginner's Guide, Academic Press, New York, 1988.

8. T. J. Richardson, Total curvature and intersection tomography, Adv. in Math., to appear.

9. L. A. Santaló, Integral Geometry and Geometric Probability, Addison-Wesley, Reading, MA, 1976.

10. S. Sherman, A comparison of linear measures in the plane, Duke Math. J., 9 (1942), 1-9.

11. H. Steinhaus, Length, shape, and area, Colloq. Math., 3 (1954), 1-13.

12. W. P. Ziemer, Weakly Differentiable Functions, Springer-Verlag, New York, 1989.

Received October 24, 1994, and in revised form September 12, 1995 